\title{
The Particle-Vibration Coupling Form Factor
}

\author{
J. Cugnon and O. Harouna \\ Institut de Physique, Université de Liège, Liège, Belgium \\ Received February 11, 1981
}

A simple model for calculating the particle-vibration form factor is studied. Explicit expressions of the latter quantity are worked out for various kinds of vibrations, assuming a static, zero range effective interaction. Numerical results for $(T=0)$ and $(T$ $=1$ ) octupole vibrations, for Fermi and Gamow-Teller vibrations are given. The influence of the range of the effective interaction is discussed in relation with the nature of the vibration.

\section{Introduction}

The interaction between a valence (or incoming) nucleon and a nuclear core seems to play an important role in various phenomena like the compression of the single-particle levels near the Fermi sea [1-3], the contraction of nuclear valence orbitals $[1,4]$ and the radiative capture of nucleons by atomic nuclei. The description of this coupling has gone in two main directions. On the one hand, one adopts a phenomenological view, assuming that the core can vibrate and that these vibrations can be described by a phonon field. An important quantity entering into this picture is the so-called form factor which essentially describes the probability of exciting the one-phonon state as a function of the position of the valence nucleon. It is usually thought that the form factor peaks at the nuclear surface, an argument based on some hydrodynamical models [5].

On the other hand, in the recent years, the microscopic description of the particle-vibration coupling has made substantial progress. In this approach, the vibrational state is viewed as a superposition of particle-hole excitations of the Hartree-Fock ground state. The probability of exciting the vibration is related to the matrix element of some effective interaction between the one-particle and the one-particle plus vibration states. This approach is very promising. In particular, it shows that the particle- vibration coupling compresses the density of the single-particle states near the Fermi surface [3]. However, it requires an important computational effort, that must be repeated for every nucleus. For the analysis of the radiative capture of nucleons or of the deep inelastic collisions between heavy ions, processes which are already quite complex, the microscopic description of the vibration is prohibited for practical reasons and one has to rely upon the phenomenological description. A good knowledge of the form factor is then desirable. We are, however, far from this goal, since at least some of the radiative capture data are analyzed (in the frame of the direct-semi-direct model) with a volume form factor $[6,7]$.

We would like to clarify the issue by a kind of intermediate approach. What we do essentially is to assume that the vibrational (giant) state exhausts the non energy weighted sum rule relative to some operator with the same quantum numbers. This permits us to construct this state in a simple manner. The next step is to write the interaction hamiltonian in a microscopic manner (i.e., with an effective nucleonnucleon interaction) and in a macroscopic form (involving the amplitude of the vibration). A comparison of the matrix elements yield the expression of the form factor. The same arguments have been advanced in $[6,8]$, but have not been developped. 
In [9], we have briefly reported on the results of the calculations we made for the dipole and quadrupole vibrations. We showed that the shape of the form factor lies in between the surface and volume forms. Our purpose here is threefold. Firstly, we want to give the derivation of the expression of the form factor, for many different vibrations, using a static zero-range interaction. We study center of mass corrections (this point was overlooked in [9]). Secondly, we report on numerical results for octupole, Fermi and Gamow-Teller vibrations. Finally, we discuss the importance of the effective interaction and indicate how our conclusions may change if one goes to more complicated effective interactions.

\section{The Model}

The interaction between an incident valence nucleon and the core can be given under the microscopic form

$H_{\mathrm{int}}=\sum_{i} v\left(x, x_{i}\right)$

where $x$ represents the space, spin and isospin coordinates for the valence nucleon and where $x_{i}$ stands for the same coordinates of the $i^{\text {th }}$ nucleon of the core. For simplicity, we will consider first a static contact interaction:

$$
\begin{aligned}
& v\left(x, x_{i}\right)=\delta\left(\mathbf{r}-\mathbf{r}_{i}\right) \\
& \cdot\left[P_{0}+P_{S} \boldsymbol{\sigma} \cdot \boldsymbol{\sigma}_{i}+P_{T} \boldsymbol{\tau} \cdot \boldsymbol{\tau}_{i}+P_{T S} \boldsymbol{\sigma} \cdot \boldsymbol{\sigma}_{i} \boldsymbol{\tau} \cdot \tau_{i}\right] .
\end{aligned}
$$

The interaction hamiltonian can also be written as a scalar product of the irreductible tensors relative to the nucleon $\left(\mathscr{M}_{\mu}\right)$ and to the core $\left(A_{\mu}(r)\right)$ :

$H_{\mathrm{int}}=\sum_{\mu} P_{\mu} A_{\mu}(r) \mathscr{M}_{\mu}$,

where $\mu$ stands for $\mu=\left[L M T M_{T} S M_{S}\right]$ and where

$$
\begin{aligned}
\mathscr{M}_{\mu} & =Y_{L M}^{*}(\Omega) \xi_{S M_{S}} \eta_{T M_{T}}, \\
A_{\mu}(r) & =\sum_{i} \frac{\delta\left(r-r_{i}\right)}{r^{2}} \xi_{S M_{S}}(i) \eta_{T M_{T}}(i) Y_{L M}\left(\Omega_{i}\right), \\
\xi_{S M_{S}} & =\eta_{T M_{T}}=1, \\
\text { for } S & =0 \text { and } T=0, \\
\xi_{S M_{S}} & =\sigma_{M_{S}}, \\
\eta_{T M_{T}} & =\tau_{M_{T}},
\end{aligned}
$$

for $S=1$ and $T=1$, respectively. The $\sigma$ and $\tau$ are the usual Pauli matrices in spin and isospin spaces. The quantity $P_{\mu}$ depends upon the indices $S$ and $T$ of the set $\mu$ only, in the case of the interaction (2.2).

Alternatively, the interaction hamiltonian can be written in the following macroscopic form:

$H_{\mathrm{int}}^{\prime}=\sum_{\mu} k_{\mu} h_{\mu}(r) \mathscr{M}_{\mu} \alpha_{\mu}$,

where $\alpha_{\mu}$ is now the collective coordinate associated with the vibration of the core, $k_{\mu}$ is the coupling constant and $h_{\mu}(r)$ is the form factor. The way we calculate $h_{\mu}$ can be described in the three following steps:

(i) For a given multipole $\mu$, we assume that the giant resonance state $\left|G_{\mu}\right\rangle$ is related to the ground state $|0\rangle$ by:

$\left|G_{\mu}\right\rangle=\frac{Q_{\mu}|0\rangle}{\left\langle 0\left|Q_{\mu}^{+} Q_{\mu}\right| 0\right\rangle^{1 / 2}}$,

where $Q_{\mu}$ is a one-body operator of multipolarity $\mu$ :

$Q_{\mu}=\sum_{i} f\left(r_{i}\right) \xi_{S M S}(i) \eta_{T M_{T}}(i) Y_{L M}\left(\Omega_{i}\right)$.

Relation (2.6) amounts to assume that the state $\left|G_{\mu}\right\rangle$ exhausts the non-energy weighted sum rule for the operator $Q_{\mu}$.

(ii) The vibration is described as a one-phonon state. More precisely, we assume that the giant resonance state is a pure one-phonon state relative to the collective variable $\alpha_{\mu}$, while the ground state is the zero-phonon state. Equivalently, we require:

$$
\left\langle G_{\mu}\left|H_{\mathrm{int}}\right| 0\right\rangle=\left\langle 1 \mu\left|H_{\mathrm{int}}^{\prime}\right| 0\right\rangle,
$$

where the states on the r.h.s. are the one-phonon and zero-phonon state respectively. From (2.3), (2.5) and (2.8), we get

$P_{\mu}\left\langle G_{\mu}\left|A_{\mu}(r)\right| 0\right\rangle=k_{\mu} h_{\mu}(r)\left\langle 1 \mu\left|\alpha_{\mu}\right| 0\right\rangle$.

(iii) We assume that $Q_{\mu}$ and $\alpha_{\mu}$ have the same matrix elements between the ground state and the giant resonance state. In other words, we assume that $Q_{\mu}$ provides a microscopic description of the collective coordinate. With the help of (2.6), we get:

$k_{\mu} h_{\mu}(r)=P_{\mu} \frac{\left\langle 0\left|Q_{\mu}^{+} A_{\mu}(r)\right| 0\right\rangle}{\left\langle 0\left|Q_{\mu}^{+} Q_{\mu}\right| 0\right\rangle}$.

Because of (2.4b) and (2.7), $Q_{\mu}$ can be written as

$Q_{\mu}=\int_{0}^{\infty} f(r) A_{\mu}(r) r^{2} d r$.

If we demand that $h_{\mu}(r)$ satisfies the normalization condition 
$\int_{0}^{\infty} h_{\mu}(r) f(r) r^{2} d r=1$

we get

$k_{\mu}=P_{\mu}$

and

$h_{\mu}(r)=\frac{\left\langle 0\left|Q_{\mu}^{+} A_{\mu}(r)\right| 0\right\rangle}{\left\langle 0\left|Q_{\mu}^{+} Q_{\mu}\right| 0\right\rangle}$.

\section{Results for Different Multipoles}

We will calculate here $h_{\mu}(r)$ using the simplest approximation, namely the Slater determinant approximation for the ground state. We start with the most studied case of the isoscalar and isovector parts of the electric multipoles. As usual for these cases, we will choose $f(r)=r^{L}$.

\section{III.1. The Isovector Multipoles}

This case correspond to $L \neq 0, M_{L}=M, S=0, T=1$, $M_{S}=M_{T}=0$. Equations (2.4b) and (2.7) become

$A_{\mu}(r)=\frac{1}{r^{2}} \sum_{i} \tau_{3 i} \delta\left(r-r_{i}\right) Y_{L M}\left(\Omega_{i}\right)$

$Q_{\mu}=\sum_{i} \tau_{3 i} r_{i}^{L} Y_{L M}\left(\Omega_{i}\right)$

The operators $Q_{\mu}^{+} A_{\mu}$ and $Q_{\mu}^{+} Q_{\mu}$ entering in (2.10) are each composed of a sum of a one-body and a two-body operators

$Q_{\mu}^{+} A_{\mu}=\sum_{i} \theta_{1}(i)+\frac{1}{2} \sum_{i \neq j} \theta_{2}(i, j)$

where

$\theta_{1}(i)=\frac{\delta\left(r-r_{i}\right)}{r^{2}} r_{i}^{L}\left|Y_{L M}\left(\Omega_{i}\right)\right|^{2}$,

$\theta_{2}(i, j)=\frac{1}{r^{2}}\left\{r_{i}^{L} \delta\left(r-r_{j}\right) Y_{L M}^{*}\left(\Omega_{i}\right) Y_{L M}\left(\Omega_{j}\right)\right.$

$\left.+r_{j}^{L} \delta\left(r-r_{i}\right) Y_{L M}^{*}\left(\Omega_{j}\right) Y_{L M}\left(\Omega_{i}\right)\right\} \tau_{3 i} \tau_{3 j}$.

Similarly,

$Q_{\mu}^{+} Q_{\mu}=\sum_{i} \zeta_{1}(i)+\frac{1}{2} \sum_{i \neq j} \zeta_{2}(i, j)$,

with

$$
\begin{aligned}
& \zeta_{1}(i)=r_{i}^{2 L}\left|Y_{L M}\left(\Omega_{i}\right)\right|^{2}, \\
& \zeta_{2}(i, j)=r_{i}^{L} r_{j}^{L}\left\{Y_{L M}^{*}\left(\Omega_{i}\right) Y_{L M}\left(\Omega_{j}\right)\right. \\
& \left.+Y_{L M}^{*}\left(\Omega_{j}\right) Y_{L M}\left(\Omega_{i}\right)\right\} \tau_{3 i} \tau_{3 j} .
\end{aligned}
$$

By using the standard techniques, we get:

$\left\langle 0\left|\theta_{1}\right| 0\right\rangle=r^{L} \rho(r)$,

where $\rho(r)$ is the nucleon core density, and

$\left\langle 0\left|\theta_{2}\right| 0\right\rangle_{\mathrm{DIR}}=\sum_{a} \sum_{b} m_{\tau_{a}} m_{\tau_{b}} \phi_{b}^{2}(r) \int \phi_{a}^{2}\left(r_{1}\right) r_{1}^{L+2} d r_{1}$

$\cdot \sum_{\substack{m_{\alpha} m_{\alpha^{\prime}} \\ m_{\beta} m_{\beta^{\prime}}}}\left(l_{a} m_{x} \frac{1}{2} m_{\alpha^{\prime}} \mid j_{a} m_{a}\right)^{2}\left(l_{b} m_{\beta} \frac{1}{2} m_{\beta^{\prime}} \cdot j_{b} m_{b}\right)^{2}(2 L+1)$

$\cdot\left(2 l_{a}+1\right)\left(2 l_{b}+1\right)(-)^{m_{\alpha}+m_{\beta}}\left(\begin{array}{ccc}l_{a} & l_{a} & L \\ m_{\alpha} & -m_{\alpha} & 0\end{array}\right)$

$\cdot\left(\begin{array}{ccc}l_{b} & l_{b} & L \\ m_{\beta} & -m_{\beta} & 0\end{array}\right)\left(\begin{array}{ccc}l_{a} & l_{a} & L \\ 0 & 0 & 0\end{array}\right)\left(\begin{array}{ccc}l_{b} & l_{b} & L \\ 0 & 0 & 0\end{array}\right)$.

For the odd multipoles, this matrix element vanishes because of the last two $3 j$-symbols. As for the even multipoles, this property is not true in general, but it holds for $L=2$, as it can be proven by direct calculation using the particular values of the $3 j$-symbols. For the exchange term, we get

$\left\langle 0\left|\theta_{2}\right| 0\right\rangle_{\mathrm{EX}}=-\sum_{a} \sum_{b} \delta_{m_{\tau_{a}} m_{\tau_{b}}} \phi_{a}(r) \phi_{b}(r)$

$\cdot \int \phi_{a}\left(r_{1}\right) \phi_{b}\left(r_{1}\right) r_{1}^{L+2} d r_{1}[J(\Omega)]^{2}$,

with

$J(\Omega)=\left[\frac{(2 L+1)\left(2 l_{a}+1\right)\left(2 l_{b}+1\right)}{4 \pi}\right]^{\frac{1}{2}}\left(\begin{array}{ccc}l_{a} & L & l_{b} \\ 0 & 0 & 0\end{array}\right)$

$\cdot \sum_{\substack{m_{\alpha} m_{\beta} \\ m_{\gamma}}}\left(l_{a} m_{\alpha} \frac{1}{2} m_{\gamma} \mid j_{a} m_{u}\right) \quad\left(l_{b} m_{\beta} \frac{1}{2} m_{\gamma} \mid j_{b} m_{b}\right)$

$\cdot\left(\begin{array}{ccc}l_{a} & L & l_{b} \\ m_{\alpha} & M & -m_{\beta}\end{array}\right)(-)^{m_{\beta}}$

Using a well-known relation between the $3 j$ - and $6 j$ symbols [10], we finally get:

$\left\langle 0\left|\theta_{2}\right| 0\right\rangle_{\mathrm{EX}}=-\sum_{[a]} \sum_{[b]} \delta_{m_{\tau_{a}} m_{\tau_{b}}} \phi_{a}(r) \phi_{b}(r)$

$\int \phi_{a}\left(r_{1}\right) \phi_{b}\left(r_{2}\right) r_{1}^{L+2} d r_{1}$

$\frac{\left(2 l_{a}+1\right)\left(2 l_{b}+1\right)\left(2 j_{a}+1\right)\left(2 j_{b}+1\right)}{4 \pi}$

$\cdot\left(\begin{array}{ccc}l_{a} & L & l_{b} \\ 0 & 0 & 0\end{array}\right)^{2}\left\{\begin{array}{ccc}j_{a} & L & j_{b} \\ l_{b} & \frac{1}{2} & l_{a}\end{array}\right\}^{2}$

The symbol $[a]$ means that the summation over the magnetic quantum numbers has already been performed. The matrix elements of $\zeta_{1}$ and $\zeta_{2}$ can be obtained by mere substitution. We gather the results 
and give the final form of $h_{\mu}(r)$

$$
h_{\mu}(r)=\frac{r^{L} \rho(r)-F_{L}(r)}{\frac{A}{4 \pi}\left\langle r^{2 L}\right\rangle-H_{L}},
$$

where $\left\langle r^{2 L}\right\rangle$ is the $2 L^{\text {th }}$ moment of the density distribution. The function $F_{L}(r)$ is given by

$$
\begin{aligned}
& F_{L}(r)=\sum_{[a]} \sum_{[b]} \delta_{m_{\tau_{a}} m_{\tau_{b}}} \overline{r_{a b}^{L}} \phi_{a}(r) \phi_{b}(r) \\
& \frac{1}{4 \pi}\left(2 l_{a}+1\right)\left(2 l_{b}+1\right)\left(2 j_{a}+1\right)\left(2 j_{b}+1\right) \\
& \cdot\left(\begin{array}{ccc}
l_{a} & L & l_{b} \\
0 & 0 & 0
\end{array}\right)^{2}\left\{\begin{array}{ccc}
j_{a} & L & j_{b} \\
l_{b} & \frac{1}{2} & l_{a}
\end{array}\right\}^{2},
\end{aligned}
$$

where we have used the short hand notation

$\overline{r_{a b}^{L}}=\int d r \phi_{a}(r) \phi_{b}(r) r^{L+2} d r$.

The value of $H_{L}$ is

$$
\begin{aligned}
& H_{L}=\sum_{[a]} \sum_{[b]} \delta_{m_{\tau_{a}} m_{\tau_{b}}}{ }^{\frac{L_{b h}^{2}}{4} \frac{1}{4 \pi}}\left(2 l_{a}+1\right)\left(2 l_{b}+1\right)\left(2 j_{a}+1\right) \\
& \cdot\left(2 j_{b}+1\right)\left(\begin{array}{lll}
l_{a} & L & l_{b} \\
0 & 0 & 0
\end{array}\right)^{2}\left\{\begin{array}{ccc}
j_{a} & L & j_{b} \\
l_{b} & \frac{1}{2} & l_{a}
\end{array}\right\}^{2} .
\end{aligned}
$$

With the help of (3.13)-(3.15), it is easy to check that $h_{u}(r)(3.12)$ is normalized as (2.12).

If the quantities $F_{L}$ and $H_{L}$ are neglected, $h_{\mu}(r)$ reduces to the so-called volume form factor, which has been used extensively in the frame of the directsemi-direct model of fast neutron radiative capture. In [9], we have calculated $h_{\mu}(r)$, using (3.12) for the dipole and the quadrupole cases and we have shown that $h_{\mu}(r)$ lies in between the so-called surface $\left(r^{L-1} \frac{d \rho}{d r}\right)$ and volume $\left(\sim r^{L} \rho\right)$ forms. This is also the case for the octupole vibrations, as indicated by Fig. 1, although our results come closer to the surface form factor. The single-particle states are calculated from the average potential constructed by Bear and Hodgson [11]. We have, however, neglected the energy dependence of the well depth for simplicity.

\section{III.2. The Isoscalar Multipoles}

For this case $(T=S=0)$, the factors containing the $m_{\tau}$ 's in (3.5) and (3.10) disappear, but it is easy to check that the final results (3.12)-(3.15) remain unchanged.

\section{III.3. The Fermi Multipoles}

So we denote the multipoles for which $M_{L}=M, S$ $=0, M_{S}=0, T=1, M_{T}=1$. Physically, they correspond to the excitation, through a $(p, n)$ reaction, to the isobaric analog state (for $L=0$ ) or to the analog giant dipole resonance (for $L=1$ ). For the operator $\tau_{+} \tau_{-i}$, it is straightforward to check that

$$
h_{\mu}(r)=\frac{r^{L} \rho_{n}(r)-F_{L}^{n}(r)}{\frac{N}{4 \pi}\left\langle r^{2 L}\right\rangle_{n}-H_{L}^{n}} .
$$

In this formula, $\rho_{n}$ is the neutron density and $\left\langle r^{2 L}\right\rangle_{n}$ is the associated $2 L^{\mathrm{th}}$ moment. The quantitives $F_{L}^{n}$ and $H_{L}^{n}$ are given (for $L=2$ and $L=$ odd) by (3.13) and (3.15) respectively, provided the factor $\delta_{m_{\tau_{a}}, m_{\tau_{b}}}$

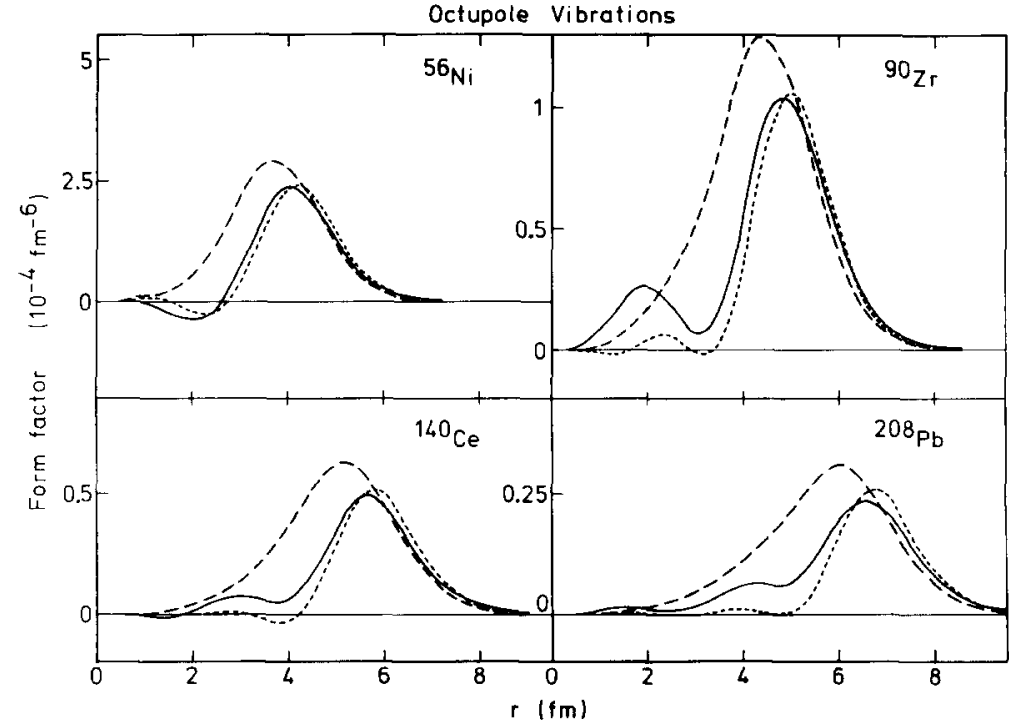

Fig. 1. Particle-vibration form factor for the $(T=0)$ nad ( $T=1)$ octupole vibrations (full curves). For comparison, the so-called volume (long dashes) and surface (short dashes) shapes are given 


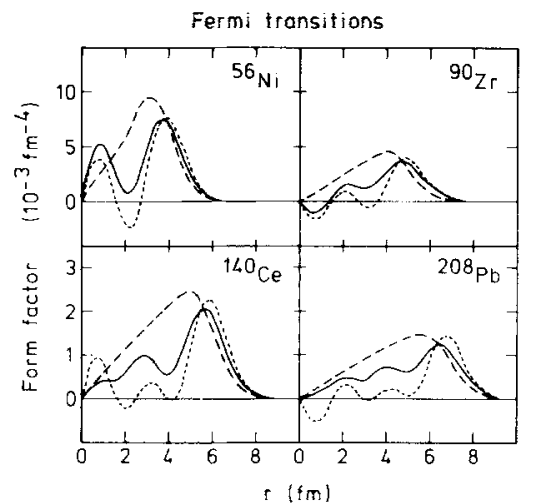

Fig. 2. Same as Fig. 1, for the $L=1$ Fermi transitions

is replaced by $\delta_{m_{\tau_{a}}, 1 / 2} \delta_{m_{\tau_{b}},-12}$. If the operator $\tau_{-} \tau_{+i}$ is considered, the results are obtained by interchanging neutrons and protons.

Let us now compare the results for $\tau_{+} \tau_{-i}, \tau_{z} \tau_{z i}$, $\tau_{-} \tau_{+i}$ for a given $L$. For $L=1$, it is easy to verify that if $N=Z$ and if the neutron and proton orbitals of identical quantum numbers are the same, all the three $h_{\mu}$ 's are the same. If $N \neq Z$ and if the orbitals are calculated in a charge-dependent mean field as usual, the $h_{\mu}$ 's are nearly equal as shown by Fig. 2 and Fig. 1 of [9].

There is an interesting feature of our model. For $L$ $=0$, direct inspection of Eqs. (3.9) and (3.10) shows that the exchange term vanishes while the direct term does not. In fact, the $L=0, \tau_{+} \tau_{-i}$ Fermi multipole corresponds to

$h_{\mu}(r)=\frac{\rho_{n}(r)-4 \pi N \rho_{p}(r)}{\frac{N}{4 \pi}-N Z}$.

In the approximation $\rho_{n}=\frac{N}{A} \rho, \rho_{p}=\frac{Z}{A} \rho$, we get

$h_{\mu}(r)=\frac{4 \pi}{A} \rho(r)$.

The same result holds for the $\tau_{z} \tau_{z i}$ and $\tau_{-} \tau_{+i}$ terms. This quantity is proportional to the density $\rho$ and can be identified to the shape of the $v_{1}$ term in the Lane model [12] (i.e. to the isovector average potential), since for these quantum numbers, the interaction (2.5) has the usual $\mathbf{t} \cdot \mathbf{T}$ form. Our calculation indicates that $v_{1}$ should be taken as constant within the nuclear volume, as it is usually done [13].

\section{III.4. The $S=1$ Multipoles}

In this case, the formulae get complicated, and the geometrical factor can only be put into a closed form if the nucleus is spin-saturated, i.e. if both the $j$ $=l+\frac{1}{2}$ and $j=l-\frac{1}{2}$ subshells are occupied for any value of $l$. For $S=1, M_{S}=0$ (the $\sigma_{z} \sigma_{z i}$ part of the interaction), it can be shown that $h_{\mu}(r)$ has still the same structure as in (3.12), with, now:

$$
\begin{aligned}
& \left\{\begin{array}{c}
F_{L}(r) \\
H_{L}
\end{array}\right\}=\sum_{n_{a} l_{a}} \sum_{n_{b} l_{b}} \delta_{\tau_{3 a} \tau_{3 b}} \frac{\left(2 l_{a}+1\right)\left(2 l_{b}+1\right)}{2 \pi} \\
& \cdot\left(\begin{array}{ccc}
l_{a} & l_{b} & L \\
0 & 0 & 0
\end{array}\right)^{2} \overline{r_{a b}^{L}}\left\{\begin{array}{ll}
\phi_{a}(r) & \frac{\phi_{b}}{r_{a b}^{L}}
\end{array}\right\} .
\end{aligned}
$$

We have implicitly assumed that the radial wave function is the same for both the $j=l+\frac{1}{2}$ and $j=l-\frac{1}{2}$ states. We have verified that the $\sigma_{+} \sigma_{-}$as well as the $\sigma_{-} \sigma_{+}$parts of the interaction yield the same result as (3.19).

It is interesting to consider the Gamow-Teller multipoles in view of the intensive experimental study of the corresponding giant resonances $[13,14]$. We now have $T=1, M_{T}=0$ or \pm 1 , and $S=1$. For the $\tau_{3} \tau_{3 i} \sigma \cdot \sigma_{i}$ part of the interaction, we get (3.12) and (3.19), since the $\tau_{3} \tau_{3 i}$ part does not introduce any difference in the two-body matrix elements (see Sect. III.1). As for the $\tau_{+} \tau_{i} \sigma \cdot \sigma_{i}$ term, we now get

$h(r)=\frac{r^{L} \rho_{n}(r)-F_{l}(r)}{\frac{N}{4}\left\langle r^{2 L}\right\rangle_{n}-H_{L}}$,

$\left\{\begin{array}{l}F_{L} \\ H_{L}\end{array}\right\}=\sum_{\substack{n_{a} l_{a} \\ \text { neutrons }}} \sum_{\substack{n_{b} l_{b} \\ \text { protons }}}\left(2 l_{a}+1\right)\left(2 l_{h}+1\right)$

$\cdot\left(\begin{array}{ccc}l_{a} & l_{b} & L \\ 0 & 0 & 0\end{array}\right)^{2} \overline{r_{a b}^{L}}\left\{\begin{array}{ll}\phi_{a}(r) & \phi_{b}(r) \\ & \overline{r_{a b}^{L}}\end{array}\right\}$.

Figure 3 shows the results of the calculation for ${ }^{40} \mathrm{Ca}$, for which we have used the latter relation and

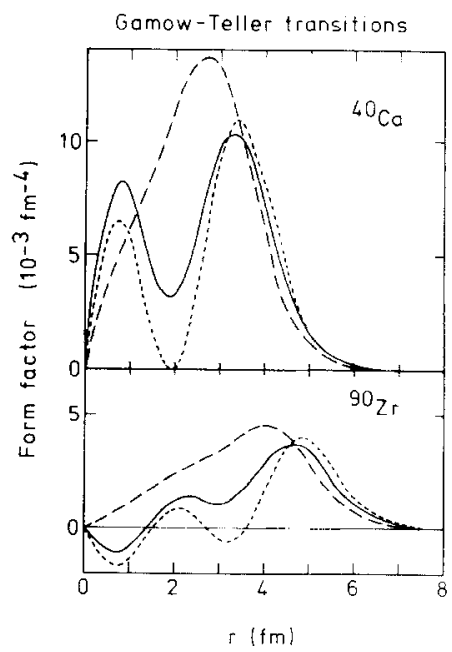

Fig. 3. Same as Fig. 1, for the $L=1$ Gamow-Teller transitions 
for ${ }^{90} \mathrm{Zr}$, where the full complexity of the expressions for $F_{L}$ and $H_{L}$ has been retained. Comparison of Figs. 2 and 3 shows that the spin operator does not practically bring any difference, and that the curves of Fig. 2 can be regarded as the Gamow-Teller form factor as well.

\section{Alternative Expressions}

We start with (2.10) and insert the identity operator as a summation over a complete set of particle-hole states between $Q_{\mu}^{+}$and $A_{\mu}$. As these are one-body operators, we may restrict ourselves to the one particle-one hole states. Furthermore, in practice, we may concentrate on the numerator only, for the normalization of $h_{\mu}$ may be obtained through (2.12). We thus have:

$h_{\mu} \sim \sum_{p, h}\left\langle\hbar\left|Q_{\mu}^{+}\right| p\right\rangle\left\langle p\left|A_{\mu}\right| \hbar\right\rangle$

where the bars indicate that the time reversed state should be taken. Of course, this expression leaves out angular momentum coupling in the particle-hole states. We give the complete formula for the simple case of $S=0$ :

$h_{\mu} \sim \sum_{(p, h) L} \frac{\left(2 j_{h}+1\right)\left(2 j_{p}+1\right)(2 L+1)}{4 \pi}\left(\begin{array}{ccc}j_{h} & L & j_{p} \\ -\frac{1}{2} & 0 & \frac{1}{2}\end{array}\right)^{2}$

$\cdot \frac{1}{2}\left[1+(-)^{l_{h}+l_{p}+L}\right] \phi_{p}(r) \phi_{h}(r)$

$\cdot \int_{0}^{\infty} \phi_{p}\left(r^{\prime}\right) \phi_{h}\left(r^{\prime}\right) r^{L+2} d r^{\prime}$

Let us mention a very peculiar fact which happens when harmonic oscillator wave functions are used. We noticed in [9] that such a choice makes $h_{\mu}$ proportional to the surface form factor, i.e. $\sim \frac{d \rho}{d r}$, for $L=1$, and $f_{1}(r)=r$ (see (2.7)). We are able to demonstrate this relation, but in order to avoid the complication of the rotational symmetry, we give the proof in one dimension only, the three dimensional extension being straightforward. We have to show that

$\sum_{p, h}\left\langle p\left|\tau_{3} z^{\prime}\right| h\right\rangle\left\langle h\left|\tau_{3} \delta\left(z-z^{\prime}\right)\right| p\right\rangle \sim \frac{d \rho}{d z}$,

where $z^{\prime}$ is the coordinate. Let us call $\phi_{1}, \ldots, \phi_{F}$ the occupied orbitals in the ground state and let us forget about the $\tau_{3}$ for the moment (we consider only one species of nucleons). We will use the property [16]

$\frac{z}{z_{0}} \phi_{n}(z)=\sqrt{\frac{n+1}{2}} \phi_{n+1}(z)+\sqrt{\frac{n}{2}} \phi_{n-1}(z)$, where $z_{0}$ is the length parameter of the harmonic oscillator. It is easy to see that the only term left in the 1.h.s. of (4.3) corresponds to $p=F+1, h=F$. Hence, we have only to show that

$\frac{d \rho}{d z} \sim \frac{1}{2} \phi_{F}(z) \phi_{F+1}(z)$.

Now,

$\frac{d \rho}{d z}=2 \sum_{n=1}^{F} \frac{d \phi_{n}(z)}{d z} \phi_{n}(z)$.

We use the well-known formula [16]

$z_{0} \frac{d \phi_{n}}{d z}=-z \phi_{n}(z)+\sqrt{2 n} \phi_{n-1}(z)$

or, using (4.4)

$z_{0} \frac{d \phi_{n}}{d z}=-\sqrt{\frac{n+1}{2}} \phi_{n+1}(z)+\sqrt{\frac{n}{2}} \phi_{n-1}(z)$.

Equations (4.8) and (4.6) yield equation (4.5). For a system of neutrons and protons, the r.h.s. of (4.5) would contain two terms, one for each species. It is interesting to note that similar relations have been developped (with an energy weighting factor) by the progenitor sum rule method [17]. To our knowledge, no similar relations exist for other multipole than the dipole one.

\section{Center of Mass Correction for the Dipole Case}

It is well known that the relevant operator, for this case, is not simply the electric dipole operator, because it contains a component along the center of mass position vector. Rather, we must use:

$Q_{\mu}=\sum_{i} g\left(\tau_{3 i}\right) \tau_{3 i} r_{i} Y_{1 M}\left(\Omega_{i}\right)$

with

$g\left(\tau_{3 i}\right)=-\frac{1-\tau_{3 i}}{2} \frac{N}{A}+\frac{1+\tau_{3 i}}{2} \frac{Z}{A}$.

This operator reduces to the isovector dipole operator (3.2) for a symmetric nucleus $(N=Z)$. As far as the form factor is concerned, there is no change, due to center of mass correction, since the operator (5.1) is a linear combination of the isovector and the isoscalar operators and since these two give rise to the same function $h_{\mu}(r)$. Note, however, that the nu- 
merator and the denominator corresponding to the operator (5.1) are not the same as for the isovector operator (3.2). This remark has some interest which is outside the scope of this work.

\section{Influence of the Effective Interaction}

We would like first to discuss briefly the sensitivity of the results under a variation of the range of the interaction. We have repeated some of the calculations (essentially for the dipole and the quadrupole cases) with a gaussian radial dependence of the effective interaction, keeping the same spin-isospin structure. We have verified that the results are practically unchanged for a range up to $0.5 \mathrm{fm}$. Beyond that, the function $h_{\mu}(r)$ keeps the same form, but its magnitude decreases (normalization (2.12) no longer applies for non-zero range forces).

The importance of the range is different from multipole to multipole and can be discussed in terms of the mass of the bosons which are thought to mediate the nuclear force. The $\tau \cdot \tau$ part of the force comes from the exchange of the $\rho$ vector meson, whose Compton wave length is equal to $0.26 \mathrm{fm}$, and hence the form factor for the corresponding multipoles is not affected by the range of the force. This is also true for the $\boldsymbol{\sigma} \cdot \boldsymbol{\sigma}$ term which comes from vector mesons exchange, except for the zero-range part of the one-pion exchange contribution. For the multipoles associated with the $\sigma \cdot \sigma \tau \cdot \tau$ term, the situation is different. This part of the interaction mainly comes from the exchange of pions, whose Compton wave length equals $1.4 \mathrm{fm}$. We have indicated in [9] that which such a range, the form factor keeps the same shape as given by our simple formula, but is renormalized by a factor $\sim 0.8$.

Of course, the properties of the effective interaction are not the same as the one of the bare nucleonnucleon force. However, it is hardly conceivable that the range of the different parts of the force is considerably changed when passing from free space into the nucleus. On the other hand, it is believed that, inside the nucleus, the nucleon-nucleon force is considerably renormalized at short distances (which is embodied by the values of the $P_{\mu}$ 's in (2.3)) and becomes density-dependent. This feature is nicely depicted by the Skyrme forces whose successes in describing static and dynamic properties are numerous [18]. Unfortunately, as for our problem, the Skyrme forces bring the complication of the velocity dependence and the particle-vibration coupling will no longer be of the simple form (2.5). If the velocity dependence of the force is neglected, it can easily be shown that the use of Skyrme force will modify our expression of $h_{\mu}(r)$ by a factor

$1+\frac{t_{3}\left(2 x_{3}+1\right)}{6 t_{0}\left(2 x_{0}+1\right)} \rho(\mathbf{r})$

where $x_{0}, x_{3}, t_{0}, t_{3}$ are the usual parameters of the Skyrme forces [18] and where $\rho(\mathbf{r})$ is the nuclear density. Consequently, $h_{\mu}$ would be reduced in the interior more than at the surface and would come closer to a surface form. To give an idea, the reduction at the interior would be of $\sim 30 \%$ for the SIII and of $\sim 20 \%$ for the SIV forces.

\section{Conclusions}

We have studied a simple model to calculate the form factor of the particle-vibration coupling hamiltonian. The model relies on a simple description of the vibrational state. This description is very likely quite accurate when the strength associated to the operator describing the collective variable is concentrated in a narrow resonance. This is certainly the case for the dipole operator in medium-heavy and heavy nuclei. This seems also to be the case for the Gamow-Teller resonance [15].

We have worked out simple formula for several interesting cases of vibrations. For $L \neq 0$ multipoles, our numerical results indicate that the form factor has a shape intermediate between the usual surface and volume forms, and this almost independently of the spin and isospin structure of the multipole operator. For the $L=0$ Fermi and Gamow-Teller operators, our model gives rise to a form factor proportional to the nuclear density. This result somehow justifies the form of the isovector term in the Lane model [12].

Our expressions hold for a static zero-range effective interaction, but we have shown that for the ordinary surface vibrations, at least, the range of the interaction does not play any significant role.

\section{References}

1. Bertsch, G.F., Kuo, T.T.S.: Nucl. Phys. A 112, 204 (1968)

2. Hamamoto, I., Siemens, P.J.: Nucl. Phys. A 269, 199 (1976)

3. Bernard, V., Nguyen Van Giai: Nucl. Phys. A 348, 75 (1980)

4. Zamick, L.: In: Common problems in low and medium energy nuclear physics. Castel, B. (ed.). New York: Plenum Press (in press)

5. Tassie, L.J.: Aust. J. Phys, 15, 135 (1962)

6. Longo, G., Saporetti, F.: Nucl. Phys. A 199, 530 (1973)

7. Saporetti, F., Fabbri, F., Guidotti, R.: Nucl. Phys. A 321, 354 (1979) 
8. Clement, C.F., Lane, A.M., Rook, J.R.: Nucl. Phys. 66, 273 (1965)

9. Cugnon, J., Harouna, O.: Phys. Lett. 91 B, 177 (1980)

10. Brink, D.M., Satchler, G.R.: Angular momentum. Oxford: Clarendon Press 1968

11. Bear, K., Hodgson, P.: J. Phys. G 4, L 287 (1978)

12. Lane, A.M.: Nucl. Phys. 35, 676 (1962)

13. Hodgson, P.E.: Nuclear reactions and nuclear structure. Chap. 9. Oxford: Clatendon Press 1971

14. Doering, R.R., Galonsky, A., Patterson, D.M., Bertsch, G.F.: Phys. Rev. Lett. 35, 1691 (1975)

15. Horen, D.J. et al.: Phys. Lett. 95 B, 27 (1980)
16. Messiah, A.: Mécanique quantique. Paris: Dunod 1960

17. Noble, J.V.: Ann. Phys. 67, 98 (1971)

18. Quentin, P., Flocard, H.: Annu. Rev. Nucl. Part. Sci. 28, 523 (1978)

\section{J. Cugnon}

Institut de Physique, B5

Université de Liège

Sart Tilman

B-4000 Liège 1

Belgium 